\title{
Ansätze für eine differenzierte Business Intelligence Governance
}

\section{Eine Konzeptentwicklung auf Basis einer Exploration}

\author{
Henning Baars, Tina Müller-Arnold, Hans-Georg Kemper \\ Lehrstubl für ABWL und Wirtschaftsinformatik I, \\ Universität Stuttgart
}

\section{Einordnung und Zielsetzung}

„Business Intelligence“ (BI) bezeichnet einen integrierten Ansatz für die betriebliche, IT-basierte Entscheidungsunterstützung (Kemper et al. 2006, S. 8). Dabei ist es vor allem der integrative Anspruch, der BI auszeichnet und aus dem die wesentlichen Herausforderungen des Gebietes resultieren - vom Aufbau anwendungsübergreifend harmonisierter Data Warehouses (DWHs) über die Zusammenführung heterogener Datenmodelle bis hin zur Implementierung einheitlicher $\mathrm{Zu}$ gangs- und Berechtigungsstrukturen. Nicht zuletzt deshalb wird die Beherrschung der Integration oftmals auch zur Bestimmung des Reifegrades eines BI-Ansatzes herangezogen (Chamoni und Gluchowski 2004, S. 119-128; Eckerson 2004, S. 3437).

In letzter Zeit ist hierbei verstärkt die organisatorische Perspektive in den Mittelpunkt gerückt. Bereits seit längerem hat sich die Erkenntnis durchgesetzt, dass für eine Entwicklung integrierter BI-Lösungen adäquate organisatorische Rahmenbedingungen und Vorgehensmodelle erfolgskritisch sind (Wixom und Watson 2001, S. 36-7). Aktuelle Ansätze gehen darüber hinaus und beziehen stärker den BI-Betrieb und die BI-Strategie mit ein (Gluchowski et al. 2008, S. 259-262). Das Gewicht derartiger Aspekte wächst dabei parallel mit dem Umfang, der Komplexität und der Bedeutung der BI-Systemlandschaften. So haben DWHs bereits in den ersten Fällen die Petabyte-Grenze durchschritten (Lai 2008), BI-Systeme mit drei bis vierstelligen Benutzerzahlen und über 11 Anwendungsbereichen sind keine Seltenheit mehr (TDWI 2009, S. 7-8; Unger und Kemper 2008, S. 146-147) und es werden die Verzahnung von dispositiven und operativen Systemen betrieben (Gluchowski et al., 2009, S. 8-12) sowie BI-Infrastrukturen aufgebaut, die über Bereichs- und Unternehmensgrenzen hinausreichen (Teradata 2007; BARC 2007, S. 20-21). 
Bestehende Organisations- und Steuerungskonzepte aus dem IT-Management sind nicht unmittelbar auf den BI-Bereich übertragbar. Gründe hierfür liegen in der im BI-Bereich besonders engen Verzahnung von fachlichen und technischen Aspekten, der hohen Änderungsdynamik sowie dem fließenden Übergang zwischen lokalen Änderungen im Rahmen des Systembetriebs und Entwicklungsprojekten mit infrastrukturellem Charakter (Moss und Shakru 2005, S. 63-80).

Als Antwort auf diese Spezifika haben viele BI-Anwenderunternehmen dedizierte BI-Organisationseinheiten eingerichtet, sogenannte Business Intelligence Competency Centers (BICCs) (Miller et al. 2006, S. 9-34; Unger und Kemper 2008). Hinsichtlich der inhaltlichen Ausgestaltung, der Dimensionierung sowie der organisatorischen Aufhängung eines BICC sind in der Unternehmenspraxis unterschiedlichste Spielarten anzutreffen, deren Konfiguration bislang nur unzureichend durch ein abgesichertes konzeptionelles Instrumentarium geleitet wird (Unger und Kemper, 2008).

Ein neuerer Ansatz ist es, den Themenkomplex systematisch von einer Governance-Perspektive zu beleuchten, die auf übergreifende Ansätze für die Gestaltung und Implementierung geeigneter Leitungs- und Kontrollstrukturen abzielt. (Gutierrez 2008; Dinter et al. 2008, S. 253-254; Horakh et al. 2008; Totok 2006, S. 63). Es ist zu betonen, dass sich die Entwicklung von Konzepten für eine so verstandene BI-Governance noch in einer frühen Phase befindet. Ziel dieses Beitrages ist es daher, auf der Grundlage einer explorativ angelegten Befragungsreihe bei BI-Anwendern ein Konzept zu entwickeln, das die Gestaltungsfelder bei der Verankerung einer BI-Governance aufzeigt und strukturiert. Ein besonderer Schwerpunkt liegt dabei auf dem Umgang mit heterogenen Rahmenbedingungen in den einzelnen Fachbereichen eines Unternehmens.

\section{Inhalte einer Business Intelligence Governance}

Ausgangspunkt der hier diskutierten Governance-Ansätze ist die so genannte Corporate Governance, die eine nachhaltige, an ethischen und kulturellen Werten ausgerichtete Unternehmensführung sicherstellen soll, indem sie geeignete Leitungs- und Kontrollstrukturen bereitstellt. Auf dieser Grundlage werden Ansätze für eine nachgelagerte, auf den IT-Bereich ausgerichtete IT-Governance entwickelt. ITGovernance befasst sich mit der Gestaltung von Regelungen, Empfehlungen, Rollen und Verantwortlichkeiten sowie mit Richtlinien zur IT-Projektpriorisierung. Sie verfolgt das Ziel, eine konsistente Ausrichtung der IT an der Strategie des Unternehmens zu gewährleisten und definiert dafür einen Rahmen für das ITManagement (Weill 2004, S. 3-4; I.T.G. Institute 2003, S. 10-12; Brown und Grant 2005, S. 688-689).

Da BI einen Teilbereich der IT darstellt, ist die IT-Governance zunächst unmittelbar für den BI-Bereich maßgeblich. Wie jedoch oben bereits ausgeführt, unterscheiden sich die Entscheidungstatbestände und Entscheidungsstrukturen in 
der BI in einigen wesentlichen Punkten von denen, die für operative IT-Systeme gültig sind. Daraus resultieren BI-spezifische Governance-Gegenstände. Genannt werden insbesondere (Dinter 2008, S. 257-258; Gutierrez 2008):

- die Gestaltung eines strategieadäquaten Portfolios von BI-Lösungen auf Basis übergeordneter BI-Prinzipien,

- die Definition BI-spezifischer Rollen, Verantwortlichkeiten und Organisationseinheiten, deren Aufhängung im Unternehmen sowie

- BI-spezifische Richtlinien, Regelungen und Empfehlungen, insbesondere bezüglich der Vorgehens- und Prozessmodelle, der durch diese gestalteten System- und Datenarchitekturen sowie hinsichtlich der zugrundeliegenden technischen Infrastruktur (Produktlandschaft).

Hierfür ist eine entsprechende BI-Governance zu konzipieren. Diese geht über die allgemeine IT-Governance hinaus, da die genannten Gegenstände an den besonderen Anforderungen der BI auszurichten sind, d.h. an dem engen Bezug zur Fachseite, an der verzahnten Natur der Infrastrukturen sowie an der hohen Änderungsdynamik. Abbildung 1 verdeutlicht die diskutierten Zusammenhänge.

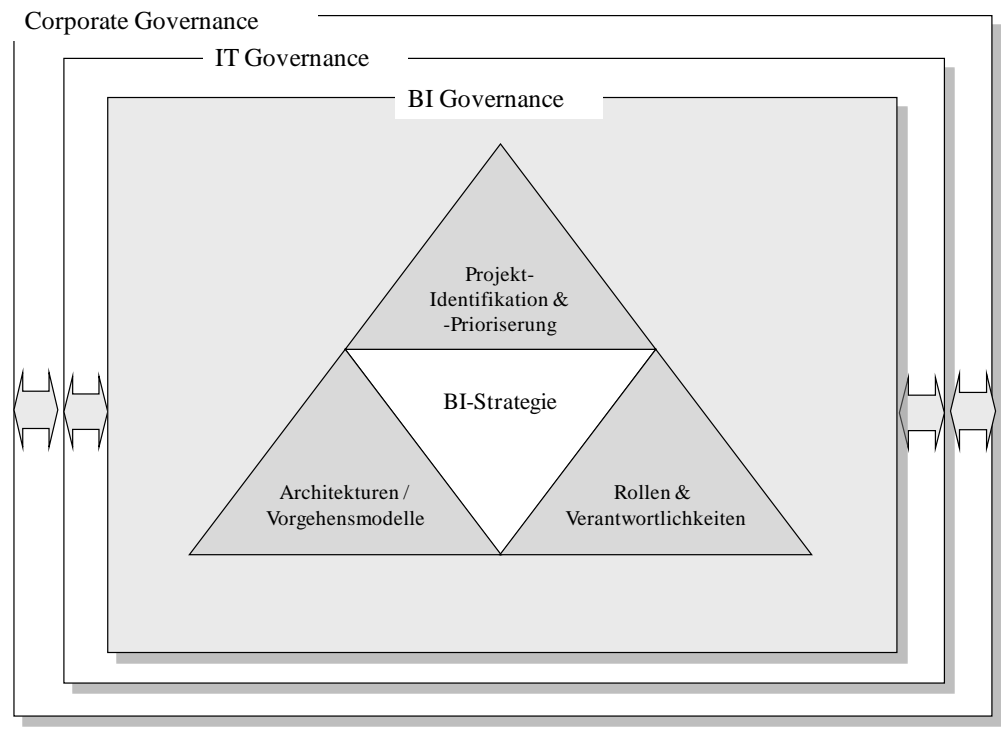

Abbildung 1: BI-Governance - Bezugsrahmen

(modifiziert übernommen von Gutierrez 2008) 


\section{BI Governance - Eine Exploration von Herausforderungen und Lösungsansätzen}

Ziel der im Folgenden vorgestellten Studie war eine qualitative Durchdringung von Herausforderungen und Lösungsansätzen im Bereich der BI-Governance. Die Ausrichtung der Untersuchungen war aufgrund der Neuartigkeit des Themenfeldes explorativ (Friedrichs 1990, S. 121). Zur Erhebung wurden leitfadengestützte, semistrukturierte Experteninterviews eingesetzt (Lamnek 1995, S. 95-110). Dem Leitfadendesign und der Ergebnisauswertung lag der in Abbildung 1 dargestellte Bezugsrahmen zugrunde, der auf der Grundlage eines Pretests mit dem Geschäftsführer eines BI-Beratungsunternehmens kalibriert wurde. Im Anschluss fanden in den Monaten September und Oktober 2008 insgesamt 8 Interviews bei BIAnwenderunternehmen mit einer Dauer von jeweils 1-2 Stunden statt (vgl. Tabelle 1). Die befragten 13 Personen wiesen einen engen beruflichen BI-Bezug auf und brachten eine mehrjährige BI-Erfahrung mit. Alle Interviews wurden transkribiert und dann systematisch anhand von Analyserastern ausgewertet. Im Weiteren werden die hier relevanten Ergebnisse diskutiert.

Tabelle 1: Übersicht über die befragten Unternehmen

Unternehmen $\mathbf{A}$ ist ein weltweit agierendes und international führendes Technologie- und Dienstleistungsunternehmen. Es besteht aus mehreren selbstständigen Geschäftsbereichen.

Unternehmen B ist ein IT-Dienstleister, der als Tochter eines internationalen Chemie- und Pharmakonzerns konzernweit IT- Dienstleistungen erbringt.

Unternehmen $\mathbf{C}$ ist ein weltweit führender Fluggesellschaftskonzern, dessen ergebnisverantwortliche Tochtergesellschaften in fünf Geschäftsfeldern zusammengefasst sind.

Unternehmen D ist ein Bankkonzern mit fünf Unternehmensbereichen unter einem gemeinsamen Vorstand mit einer zentralen IT-Organisation.

Unternehmen $\mathbf{E}$ ist die Tochter eines großen bundesweit tätigen Versicherungskonzerns.

Unternehmen $\mathbf{F}$ ist ein international ausgerichteter Chemiekonzern mit mehreren Tochterunternehmen in Deutschland.

Unternehmen $\mathbf{G}$ ist ein internationaler Konzern, dem vier dezentral organisierte Geschäftsbereiche angehören. Für die strategische und finanzielle Führung ist eine Holding zuständig, die Grundsätze und Leitlinien für alle dezentralen Geschäftsbereiche definiert.

Unternehmen $\mathbf{H}$ ist ein weltweit agierender Kraftfahrzeughersteller, der mehrere Geschäftsfelder unter einem Dach vereint. 


\subsection{Rollen und Verantwortlichkeiten}

Eine wesentliche Erkenntnis aus den Interviews ist, dass die Aufgabenabgrenzung zwischen IT und Fachbereich in den Unternehmen nicht einheitlich definiert wird, sondern je nach Fach- und Anwendungsbereich variiert: Selbst in den drei Fällen, in denen eigentlich eine klare Trennung der Aufgaben vorgesehen war (Fälle A, D und $\mathrm{H}$ ), wurde auf Ausnahmetatbestände hingewiesen. Die konkrete Arbeitsteilung ist abhängig vom IT-Know-how der jeweiligen Benutzer, von der Verfügbarkeit personeller Ressourcen, von der Dynamik im Anwendungsfeld sowie von den betroffenen Inhalten. Hierbei wird den Controlling-Abteilungen in allen befragten Unternehmen ein besonders großer Freiraum bei der Gestaltung und Nutzung der Lösungen gewährt - was mit dem in diesem Bereich hohen BI-Akzeptanz- und Nutzungsgrad erklärbar ist (BARC 2008, S. 8; Kemper und Pedell 2008, S. 32f.).

Eine flexible Aufgabenabgrenzung wird auch ausdrücklich von den Fachbereichen gefordert, da sie als Budgetgeber selbst darüber entscheiden möchten, welche Aufgaben sie durchführen dürfen und welche sie an die IT abgeben (entsprechende Aspekte wurden in den Interviews A, C, F und G angeführt). Unternehmen F verfolgt diesbezüglich das explizite Ziel, dem Fachbereich einen möglichst hohen Handlungsspielraum zu ermöglichen, wofür der Aufbau eines BI-KompetenzCenters im Fachbereich als Voraussetzung erachtet wird. Auch bei Unternehmen E wird die Auffassung vertreten, dass die IT-Abteilung im Sinne einer ,agilen $\mathrm{BI}^{\prime \prime}$ nur noch an den Stellen eingreifen und Vorgaben machen sollte, an denen der Fachbereich einen Mehrwert erhält. Entsprechende Ansätze können durch flexible Systeme unterstützt werden, wie das Beispiel von Unternehmen F zeigt: Die von F bereitgestellte Infrastruktur sieht eine „BI-Selbstbedienung“ auf Basis von „BI self services" vor. Diese ermöglichen Systemänderungen, ohne dass eine Einschaltung des ansonsten üblichen, langwierigen Anforderungsprozesses der IT notwendig wird.

Trotz entsprechend entgegenkommender Ansätze wurde speziell in den Interviews $A, B, F$ und $G$ das Spannungsfeld zwischen einer zentralen Regulierung und lokalen, fachlich getriebenen Initiativen sichtbar, auf das auch bereits in anderen Publikationen hingewiesen wurde (Eckerson und Sherman 2008; Baars et al. 2009). Hierzu eine Aussage aus Fall G:

„Die Regelung des Spannungsfelds ist mühselig, da man immer gegen einzelne Interessen verstößt. Das liegt aber bei BI in der Natur der Sache: Wenn sie ein vernünftiges Datenmodell haben wollen, dann muss jeder Kompromisse machen [...]."

Ein besonderer Konfliktherd ist die Entstehung lokaler BI-Lösungen mit fachbereichsspezifischen Datensilos („Schatten-DWHs“). Auffallend ist, dass das Phänomen bei nahezu allen Unternehmen beklagt wird (Ausnahme: Unternehmen H) - obwohl diesem aktiv entgegengewirkt wird. Schatten-DWHs völlig zu verhindern ist nach Ansicht der Befragten schwierig, da die Fachbereiche die Budgethoheit für BI-Projekte haben. Das folgende Zitat aus Interview F illustriert die Problematik: 
"[Die Fachbereiche] haben eine Fragestellung im Marketing-oder Vertriebsbereich und die müssen sie lösen. Dann gehen sie ₹u einem Beratungshaus und bekommen dort eine Lösung für ibr Problem, ₹. B. mit Hilfe von bestimmten Reports, Analysen oder Vorschlägen, welche Daten bierfür benötigt werden. Nebenbei bekommen sie dann auch noch eine Software an die Hand, so dass das Problem komplett fertig gelöst wird. D.h. der Fachbereich hat eine Fragestellung, will diese lösen und kauft eine BI-Lösung. So würde ich sagen, sind $80 \%$ der Fachbereichslösungen bier rein gekommen - und natürlich vollkommen an der IT vorbei."

In den Interviews A, C, G und F wurde eine Reihe von Ansätzen zur Vermeidung von Schatten-DWHs genannt, die im Wesentlichen auf die proaktiven Bereitstellung eines attraktiven, innovativen BI-Angebotes durch die IT hinauslaufen, das auch Beratungs- und Managementleistungen einschließt. Tendenziell beobachten die Unternehmen, dass die Entstehung von Schatten-DWHs in den Fachbereichen rückläufig ist (v. a. in den Fällen A, F, G). Dies wird bei Unternehmen G u. a. auch darauf zurückgeführt, dass ein Betrieb lokaler BI-Lösungen mit der zunehmenden Dynamik im Anwendungsumfeld an Attraktivität verliert und ein professionelles Angebot der IT zunehmend als Entlastung wahrgenommen wird.

Der bereichsübergreifenden Abstimmung kommt überdies gemäß der Interviews A, $\mathrm{B}, \mathrm{C}, \mathrm{G}$ und $\mathrm{H}$ zugute, dass die Bedeutung eines übergreifenden Know-HowAufbaus erkannt wird. Da BI oftmals sehr spezifisches Wissen verlangt, wird Know How zum Engpass, dem mit einem (in den einzelnen Fällen unterschiedlich stark institutionalisierten) Erfahrungsaustausch sowie bei drei Unternehmen auch mit einem Austausch von Mitarbeitern begegnet wird. Trotzdem sind in nahezu allen Fällen auch externe Mitarbeiter eingebunden, um verbleibende Know-HowLücken zu schließen.

Als eigene - im Leitfaden eigentlich nicht explizit abgefragte - Hauptaufgabe der BI-Governance wurde bei fünf Unternehmen (B, C, E, F, G) die Sicherstellung der Datenqualität gesehen, wobei ein akuter Handlungsbedarf bei der Installation eines Datenqualitätsverantwortlichen (Data Owner / Data Steward) verortet wird, der sowohl für technische wie auch für fachliche Fragen zuständig ist. Diese Ergebnisse unterstreichen die zentrale Bedeutung des Datenqualitätsthemas (Kemper und Pedell 2008, S. 31f.; Baars et al 2009, S. 5f.).

Schließlich wiesen die beiden Unternehmen A und F auf die Einrichtung von hochrangig besetzten BI-Steering-Committees hin, mit denen die Durchsetzung und die Akzeptanz von BI-Entscheidungen gefördert werden soll.

\subsection{Projektidentifikation und -priorisierung}

In allen Unternehmen ist die Priorisierung von BI-Anforderungen bzw. von BI-Projekten in das klassische IT-Anforderungsmanagement integriert. Abgesehen von Compliance-bedingten Auflagen, deren Umsetzung keinen Aufschub erlaubt, erfolgt hierbei in den meisten Fällen zunächst eine Priorisierung der Anforderungen durch den Fachbereich selbst (Fälle B, C, D, E, F und G). Anschließend wird diese von der 
IT auf die resultierenden Realisierungsanforderungen geprüft. Es ist wenig überraschend, dass bei einem solchen Vorgehen fachbereichsübergreifende oder gar strategische Zusammenhänge schnell vernachlässigt werden, weshalb die Befragten die Implementierung einer übergreifenden Anforderungspriorisierung als besonders notwendig erachten.

Ein in den Fällen B, E, G und $\mathrm{H}$ betriebener Ansatz ist die Etablierung von lokalen BI-Kompetenzzentren in den Fachbereichen, die u. a. auch eine unternehmensweite Abstimmung der Prioritäten mitverantworten. Darüber hinaus wurde auch die Einrichtung eines zentralen BICCs als förderlich angeführt - eine Aussage, die auch durch die Ergebnisse anderer Studien gestützt wird (BARC 2008, Baars et al. 2009).

Nur in zwei Unternehmen (A und $\mathrm{H}$ ) wird eine strategisch orientierte BIRoadmap in den Priorisierungsprozess einbezogen. Ein solches Vorgehen erleichtert insbesondere die Bewertung bereichsübergreifender Anforderungen etwa in der Datenhaltung.

\subsection{Richtlinien / Regelungen / Empfehlungen}

Die Analyse der Interviews zeigt, dass Richtlinien, Regeln und Empfehlungen v. a. in Form von Standards und Methoden auf Produktebene zum Einsatz kommen. Wie auch in anderen Publikationen dokumentiert (z. B. Horakh et al. 2008, S.4-6) hat ein unkoordinierter Produkteinsatz insbesondere Folgen für die Betriebseffizienz:

"Wir haben eine Analyse [...] gemacht und hierbei haben wir 27 verschiedene BIAnwendungen gezäblt. Dies ist ungefähr die Hälfte des repräsentativen Marktes hier in Deutschland. Wir haben vorher schon immer gesehen, dass unser Unternehmen oftmals als Referenz bei Beratungshäusern oder Herstellern genannt wurde. Nun wissen wir, es stimmt." (Aussage aus Interview F)

Im Hinblick auf den Einsatz von BI-Prozessstandards und -Methoden ist der Umgang mit Standards und Methoden im BI-Betrieb bereits gängig. Hierfür kommen überwiegend IT-Service-Management-Konzepte zum Einsatz, v. a. auf Basis des Frameworks ITIL und unter Verwendung von SLAs (eingesetzt in den Fällen A, B, C und D).

Die Regulierung der BI-Entwicklungsprozesse ist im Gegensatz dazu nur schwach ausgeprägt. Interessant ist dabei die Tatsache, dass zwar kein Unternehmen die Notwendigkeit eines Einsatzes BI-spezifischer Vorgehensmodelle sieht, jedoch ausnahmslos alle Befragten der Ansicht sind, dass ihr generelles ITVorgehensmodell flexibel anpassbar sein muss, um eine kontextabhängige Ausgestaltung zu ermöglichen. BI-spezifische Aspekte betreffen in diesem Zusammenhang v. a. die Durchführung von Informationsbedarfsanalysen, die Ausgestaltung von ETL-Prozessen sowie Anforderungen an die Datenqualität. Für letzteres werden bei allen 8 Unternehmen auch die Entwicklung und Einhaltung von datenberogenen Richtlinien und die unternehmensweite Verwendung eines einheitlichen Modellierungstools bei der BI-Entwicklung als besonders wichtig erachtet. 


\section{Ein Konzept für eine differenzierte BI-Governance}

Die Ergebnisse der Untersuchung verdeutlichen, dass es für größere BIInstallationen nicht sinnvoll ist, undifferenzierte Regelungsstrukturen aufzubauen, die unternehmensweit starr durchgesetzt werden. Zum einen sind ungewollte Regelungen im BI-Bereich politisch schwer durchsetzbar, zum anderen können sie auch besonders leicht umgegangen werden - durch Parallelstrukturen. Selbst die eigentlich elementare Forderung zur Durchsetzung einheitlicher Produktstandards wird in der Realität oftmals bewusst oder unbewusst von Anwenderseite torpediert.

Sowohl bei der Aufgabenabgrenzung und Rollendefinition, bei der Architekturgestaltung (z. B. durch punktuelle oder temporäre Duldung dezentraler Strukturen) als auch bei der Ausgestaltung der zugehörigen Vorgehensmodelle sind ein Entgegenkommen auf die Fachabteilungen sowie eine flexible Ausgestaltung der Governance-Rahmenbedingungen vonnöten.

Dies heißt aber nicht, dass auf eine übergreifende Governance verzichtet werden kann - im Gegenteil. Die Ergebnisse unterstreichen erneut die Gefahren eines dezentralen Wildwuchses. Im Folgenden wird deshalb ein Konzept vorgestellt, das auf dieses Spannungsfeld eingeht. Hierfür wird der in Abschnitt 2 dargestellte Bezugsrahmen um Komponenten für die Ausgestaltung lokaler BI-Umgebungen auf Basis eines definierten Governance-Modul-Sets erweitert. Darüber hinaus wird die aufbauorganisatorische Trägerschaft für die BI-Governance verankert. Abbildung 2 visualisiert das Konzept.

Trotz aller Tendenzen zur Entwicklung unabgestimmter Eigenlösungen legen es die Ergebnisse dennoch nahe, dass der Anwenderseite die Notwendigkeit einer übergreifenden Koordination sehr wohl erfolgreich kommuniziert werden kann. Des Weiteren wurde in den Interviews berichtet, dass schon aus ressourcenbedingten Zwängen eine zunehmende Abstimmungsbereitschaft vorliegt. Es wird vorgeschlagen, diese durch übergreifende Strukturen aufzufangen. Als solche kommen insbesondere ein BICC, matrixartig in die Fachbereiche eingewebte Kompetenzzentren sowie übergreifende Steuerungsgremien in Frage.

Mit entsprechenden Organisationseinheiten werden unmittelbare Träger für die BI-Governance geschaffen. Eine besondere Rolle kommt hierbei einem nahe an der Leitungsebene aufgehängten BI-Governance-Committee zu, das u. a. die Legitimation und Kommunikation übergreifender BI-Regulierungen fördert.

Für den Aufbau eines zentralen BICCs sowie bedarfsweise dezentraler BIKompetenzteams spricht darüber hinaus die Notwendigkeit, an der Schnittstelle zum Anwender fachliche und technische Kompetenzen zusammenzubringen. Die Ergebnisse zeigen, dass es erforderlich ist, gegenüber dem Fachbereich als aktiver Berater und Lösungsanbieter aufzutreten, der gegen interne wie externe Konkurrenz bestehen kann. Mit derartigen Rollenträgern wird es auch einfacher, den Governance-Rahmen für einen Fachbereich auszuloten. 


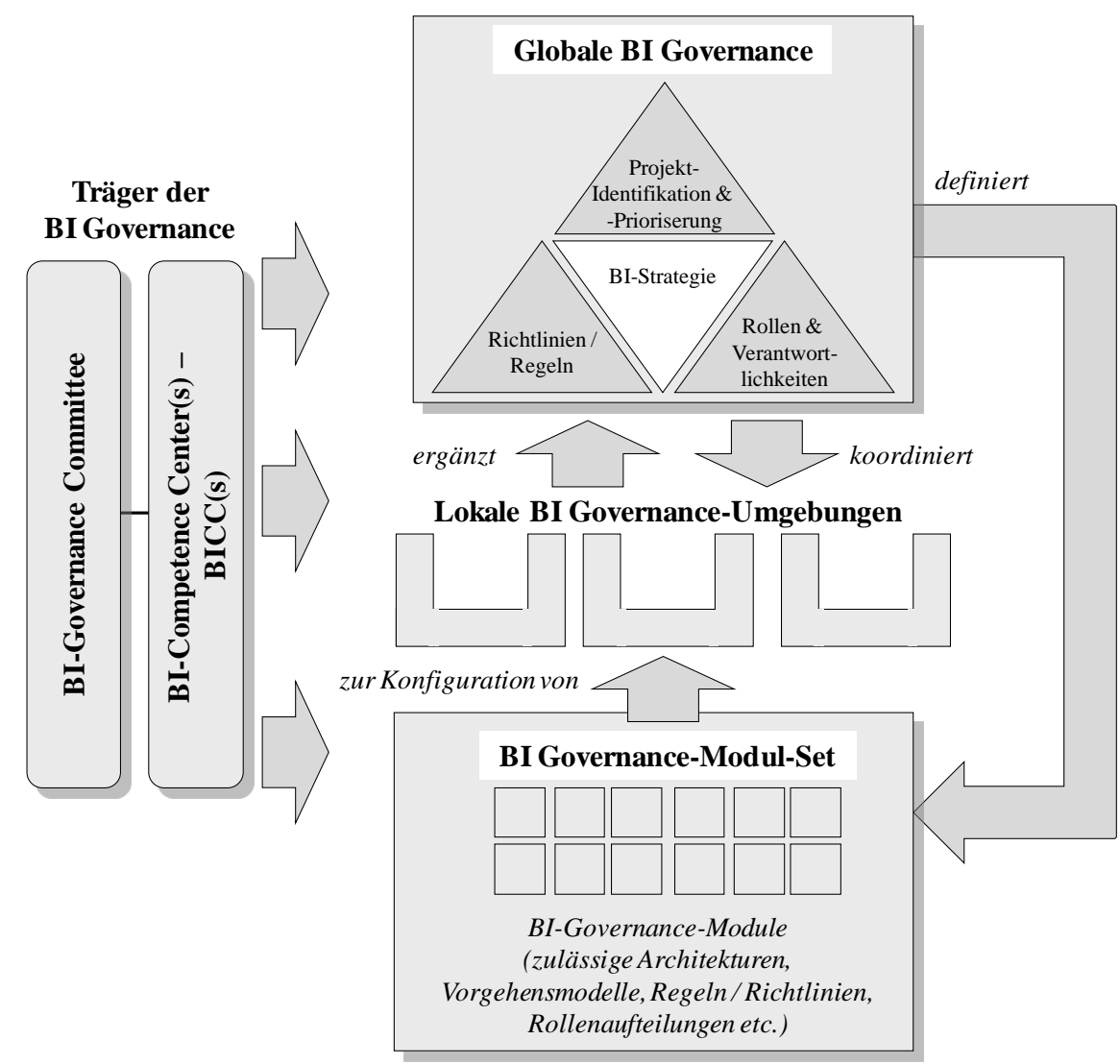

Abbildung 2: Konzept für eine differenzierte BI-Governance

Unter Berücksichtigung der spezifischen Rahmenbedingungen sowie der inhaltlichen Verflechtungen des Fachbereichs ist die BI-Governance jeweils fachbereichsindividuell auszuformen. So kann es auf Architekturseite beispielsweise sinnvoll sein, separate DWHs für unabhängige Geschäftsbereiche in einer Finanzholding, isolierte Data Marts für abgegrenzte und einmalige Analyseaufgaben oder temporärere Datenhaltungen für die zeitnahe Umsetzung von Compliance-Anforderungen zuzulassen. Liegen hingegen starke Leistungsverflechtungen zwischen Anwendungsbereichen vor, so müssen dysfunktionale Parallelstrukturen selbstverständlich umfassend abgefangen werden. Anders als teilweise gefordert, ist jedoch ein „Enterprise DWH“ nicht in jedem Fall ein erstrebenswertes Langfristziel.

Mit den Architekturen sind auch die Vorgehensmodelle und Richtlinienbündel zu differenzieren - von einem dezentralen, agilen Compliance-Projekt bis hin zum durchstrukturierten Entwicklungsprozess für eine größere Reportinglösung. Insgesamt werden so lokale BI-Governance-Umgebungen aufgebaut. Diese flankieren und ergänzen weiterhin bestehende, globale Regelungs- und Steuerungsstrukturen. 
Trotz aller Differenzierungen können dennoch Standardisierungspotentiale freigesetzt werden - sofern die entsprechenden Module hierfür übergreifend abgestimmt werden. Das in den Interviews aufgebrachte Bild der „BI-Selbstbedienung“ bietet hier einen Ansatzpunkt: Die Komponenten, aus denen die BI-GovernanceUmgebungen aufgebaut werden, können sehr wohl unternehmensweit standardisiert werden. So ist beispielsweise eine Vorgabe des Spektrums gültiger Architektur- und Vorgehensmodelle (inklusive Richtlinien zu deren Einsetzbarkeit und Ausgestaltung) und der Bandbreite an Produktanbietern sinnvoll. Ein so verstandenes Governance-Modul-Set mit aufeinander abgestimmten Regulierungsbausteinen ist kontinuierlich zu pflegen und bei Bedarf zu erweitern.

Unabhängig davon ist durch die beschriebenen Gremien das Gesamtportfolio an Lösungen zu verfolgen und strategieorientiert weiterzuentwickeln. Eine rein fachseitig betriebene Priorisierung ist hierfür unzureichend. Es muss vielmehr die Gesamtinfrastruktur überwacht und dabei eine strategiekonforme Entwicklung der „Architektur aus Architekturen“ betrieben werden. Nur so lässt sich sicherstellen, dass die Managementunterstützung dem im BI-Begriff enthaltenen Integrationsanspruch auch gerecht wird.

\section{Diskussion und Ausblick}

Organisatorische, technische und fachliche Gründe sprechen dafür, dass eine Differenzierung lokaler BI-Governance-Umgebungen den betrieblichen Realitäten von Unternehmen mit einer ausgeprägten BI-Nutzung eher gerecht wird als Ansätze, die stark auf einheitliche Vorgaben setzen. Zu stark unterscheiden sich die Rahmenbedingungen bei den Fachbereichen, als dass Architekturen, Vorgehensmodelle, Rollenabgrenzungen und Richtlinien vollständig vereinheitlicht werden könnten. Das gilt vor allem für größere, in Konzernstrukturen organisierte Unternehmen, wie die in der Studie betrachteten.

Es besteht jedoch noch Konkretisierungsbedarf hinsichtlich der unternehmensspezifischen Ausgestaltung des Ansatzes. Insbesondere sind die Rahmenbedingungen zu spezifizieren, unter denen bestimmte Architekturen, Vorgehensmodelle und Regelungen zulässig und sinnvoll sind, um somit Hilfestellung beim Aufbau des Governance-Modul-Sets zu liefern. Darüber hinaus ist das Konzept in einem nächsten Schritt mit den ebenfalls bereits in einigen Unternehmen etablierten BI-Service-Ansätzen in Bezug zu setzen. Wie das Beispiel von Unternehmen F nahe legt, wird die Durchsetzung von Governance-Strukturen erleichtert, wenn diese über Systemtemplates und/oder definierte Servicekomponenten in die Anwendungsbereiche getragen wird. Entsprechend sind die Abhängigkeiten zur ITGovernance und zum IT-Servicemanagement zu konkretisieren und flankierende Kennzahlenkonzepte auszuarbeiten.

Insgesamt ist davon auszugehen, dass dem Bereich BI-Governance mit der Verbreitung unternehmensübergreifender BI-Lösungen sowie im Zuge komplexi- 
tätstreibender Trends wie der Virtualisierung von BI-Infrastrukturen oder dem „Cloud Computing“ in Zukunft noch deutlich mehr Gewicht zukommen wird.

\section{Literatur}

Baars H, Zimmer M, Kemper HG (2009) The Business Intelligence Competence Centre as an Interface between IT and User Departments in Maintenance and Release Development. Proceedings of The 17th European Conference on Information Systems (ECIS 2009), 08.-10.06.2009, Verona

BARC (2007) Klassenbester - Convenience-Stores mit Business Intelligence. In: BARC-Guide Busienss Intelligence 2006/2007. Würzburg, S. 20-21.

BARC (2008) Organization of Business Intelligence. Würzburg.

Brown AE, Grant GG (2005) Framing the Frameworks: A Review of IT Governance Research. In: Communications of the Association for Information Systems 15 (38), 696-712.

Chamoni P, Gluchowski P (2004) Integrationstrends bei Business-IntelligenceSystemen - Empirische Untersuchung auf Basis des Business Intelligence Maturity Model. In: Wirtschaftsinformatik 46 (2), S. 119-128.

Dinter B, Lahrmann G, Meyer D, Schmaltz M (2008) Governance in der Informationslogistik am Beispiel eines Energieversorgers. In: Dinter B, Winter R, Chamoni P, Gronau N, Turowski K (Hrsg.) Tagungsband der DW 2008: Synergien durch Integration und Informationslogistik, S. 249-266.

Eckerson W (2004) Gauge Your Data Warehousing Maturity. In: DM Review, S. 34-37. http://www.information-management.com/issues/20041101 /1012391-1.html (November 2004). Abgerufen am 20.09.2009.

Eckerson W, Sherman RP (2008) Strategies for Managing Spreadmarts. In: Business Intelligence Journal 13 (1), S. 23-24.

Friedrichs J (1990), Methoden empirischer Sozialforschung, 14. Auflage. Westdeutscher, Opladen.

Gluchowski P, Gabriel R, Dittmar C (2008) Management Support Systeme und Business Intelligence - Computergestützte Informationssysteme für Fach- und Führungskräfte, Springer, Berlin.

Gluchowski P, Kemper HG, Seufert A (2009) Innovative Prozess-Steuerung Was ist neu an Operational BI? In: BI-Spektrum, 4 (1), S. 8-12.

Gutierrez N (2008) Business Intelligence (BI) Governance, http://www.businessintelligence.com/article.asp?id=170. Abgerufen am 30.10.2008. 
Horakh T, Baars H, Kemper HG (2008) Mastering Business Intelligence Complexity - A Service-based Approach as a Prerequisite for BI Governance. In: Proceedings of the $14^{\text {th }}$ Americas Conference on Information Systems (AMCIS), Toronto, Canada.

I. T. G. Institute, (2003) Board Briefing on IT Governance. IT Governance Institute, Rolling Meadows(USA).

Kemper HG, Mehanna W, Unger C (2006) Business Intelligence - Grundlagen und praktische Anwendungen eine Einführung in die IT-basierte Managementunterstützung, 2. Aufl. Vieweg, Wiesbaden.

Kemper HG, Pedell B (2008) BI im Controlling - eine Bestandsaufnahme. In: BI Spektrum 3 (3), S. 28-33.

Lai E (2008) Teradata creates elite club for petabyte-plus data warehouse customers, Computerworld, http:/ /www.computerworld.com / action/ article.do? command $=$ viewArticleBasic\&articleId=9117159. Veröffentlicht am 14.10.2008, abgerufen am 28.04.2009.

Lamnek S (1995) Qualitative Sozialforschung, 3. Aufl. Beltz, Basel.

Miller GJ, Bräutigam D, Gerlach SV (2006) Business intelligence competency centers: a team approach to maximizing competitive advantage. Wiley, Hoboken (USA).

Moss, ST, Shakru A (2005) Business Intelligence Roadmap - The Complete Project Lifecycle for Decision Support Applications. Wiley, Boston, USA..

TDWI (2009) 2009 TDWI BI Benchmark Report. TDWI, Renton, USA.

Teradata (2007) Enterprise Data Warehouse Delivers Healthy Returns to BKK Insurance Companies, Teradata Whitepaper, S. 11.

Totok A (2006) Entwicklung einer Business-Intelligence-Strategie. In: Chamoni P, Gluchowski, P (Hrsg.) Analytische Informationssysteme, 3. Aufl., Springer, Berlin, Heidelberg, S. 51-70.

Unger C, Kemper HG (2008) Organisatorische Rahmenbedingungen der Entwicklung und des Betriebs von Business Intelligence - Ergebnisse einer empirischen Studie. In: Bichler M, Hess T, Krcmar H, Lechner U, Matthes F, Picot A, Speitkamp B, Wolf P (Hrsg.) Tagungsband der Multikonferenz Wirtschaftsinformatik 2008. München, S. 141-153.

Weill P (2004). Don't Just Lead, Govern: How Top-Performing Firms Govern IT. In: MIS Quarterly Executive, 3 (1), 1-17.

Wixom BH, Watson HJ (2001). An empirical investigation of the factors affecting data warehousing success. In: MIS Quarterly, 25 (1), 17-41. 